\title{
Efeito da estimulação elétrica na cicatrização de feridas cutâneas em Wistar: existe protocolo a ser seguido? (Revisão de estudos experimentais)
}

\author{
Electric stimulation uses on cutaneous wound healing: A literature review of experimental studies
}

in Wistar rats

Efecto de la estimulación eléctrica sobre la curación de heridas cutáneas en Wistar: ¿hay algún protocolo a seguir? (Revisión de estudios experimentales)

\author{
Ailton Mota do Nascimento Galvão \\ ORCID: https://orcid.org/0000-0002-9268-6921 \\ Universidade Federal de Alagoas, Brasil \\ E-mail: ailton_fisioterapeuta@ hotmail.com \\ Zenaldo Porfirio da Silva \\ ORCID: https://orcid.org/0000-0002-6970-4474 \\ Universidade Federal de Alagoas, Brasil \\ E-mail: seisporfirio@gmail.com \\ Ana Paula Fernandes Barbosa \\ ORCID: https://orcid.org/0000-0002-5835-0359 \\ Universidade Estadual de Ciências da Saúde de Alagoas, Brasil \\ E-mail: npdc@uol.com.br \\ Célio Fernando de Sousa Rodrigues \\ ORCID: https://orcid.org/0000-0002-1361-8139 \\ Universidade Federal de Alagoas, Brasil \\ E-mail:celiofernando@yahoo.com.br
}

\begin{abstract}
Resumo
A Estimulação Elétrica tem mostrado ter efeitos benéficos no processo de cicatrização de feridas de pele em ratos Wistar. É importante avaliar os efeitos da eletroestimulação na reparação de feridas a fim de garantir a otimização de sua prática. Objetivo: Este artigo de revisão consiste em avaliar a resposta cicatricial em ratos Wistar, tratados com a microcorrente. Material e método: Realizou-se uma busca nas bases de dados eletrônicas PubMed no período entre 1966 e janeiro 2021, Lilacs entre 1982 e janeiro 2021 e SciELO, utilizando-se os descritores "Electric Stimulation", "Wound Healing" e "Wistar", os quais foram selecionados segundo os Descritores em Ciências da Saúde. Os critérios de inclusão consistiram em estudos com animais wistar, tratados com eletroestimulação e publicados nas línguas inglesa ou portuguesa. Resultados: Foram selecionados seis estudos, os quais sugeriram que a corrente elétrica pode estimular a cicatrização, crescimento e a regeneração. Observou-se uma pequena quantidade de artigos relacionados à estimulação elétrica no processo cicatricial de feridas, bem como falta de padronização nos parâmetros utilizados nesses artigos, o que ainda impossibilita uma revisão sistemática. Também é consensual a preocupação com a necessidade de uniformização das doses utilizadas, visto que cada autor defende a sua aplicabilidade. Conclusão: Apesar da falta de padronização dos parâmetros para o tratamento de feridas em geral com estimulação elétrica em ratos Wistar, as análises morfológica e histológica, mostram uma resposta eficaz no reparo tecidual nas feridas submetidas às aplicações das microcorrentes nos diferentes períodos de tratamento.
\end{abstract}

Palavras-chave: Estimulação; Cicatrização de feridas; Wistar.

\begin{abstract}
The Electric Stimulation (ES) has been shown to have beneficial effects in wound healing. It is important to assess the effects of ES on the healing of skin wounds in order to guarantee the optimal clinical practice. Objective: This review article aimed to evaluate the importance of ES in wound healing by the scarce literature and the lack of consensus among the treatments. Methods: A search in the electronic databases PubMed, Lilacs and SciELO was performed using the keywords "Stimulation", "wound healing" and "Wistar". Inclusion criteria consisted of studies with Wistar animals treated with ES, published in English or Portuguese languages in the period between 1966 and 2021 . The data from the study results were determined by morphology. Results: seven studies were selected and all pointed to the importance of the electrical stimulation mode to accelerate wound healing. Although there is a considerable amount of research on the effect of ES as an adjunct in the treatment of various pathologies, a small amount and a great variability in the parameters used is observed in the healing process. There is also a consensual concern about the need for standardization of doses, as each author defends its applicability. Best results are achieved when using the
\end{abstract}


micro amperage isolated or associated in the treatment of wound healing. Conclusion: Further studies are needed to corroborate with the padronization of the electrical stimulation in the cutaneous wound healing process.

Keywords: Stimulation; Wound healing; Wistar.

\begin{abstract}
Resumen
Se ha demostrado que la estimulación eléctrica tiene efectos beneficiosos sobre el proceso de curación de heridas en la piel en ratas Wistar. Es importante evaluar los efectos de la estimulación eléctrica en la reparación de heridas para asegurarse de que su práctica esté optimizada. Objetivo: Este artículo de revisión es evaluar la respuesta de curación en ratas Wistar tratadas con microcorriente. Material y método: Se realizó una búsqueda en las bases de datos electrónicas PubMed entre 1966 y enero de 2021, Lilacs entre 1982 y enero de 2021 y SciELO, utilizando los descriptores "Estimulación eléctrica", "Cicatrización de heridas" y "Wistar", los cuales fueron seleccionados según a los Descriptores de Ciencias de la Salud Los criterios de inclusión consistieron en estudios con animales Wistar, tratados con estimulación eléctrica y publicados en inglés o portugués. Resultados: Se seleccionaron seis estudios que sugirieron que la corriente eléctrica puede estimular la curación, el crecimiento y la regeneración. Hubo un pequeño número de artículos relacionados con la estimulación eléctrica en el proceso de cicatrización de heridas, así como una falta de estandarización en los parámetros utilizados en estos artículos, lo que aún imposibilita una revisión sistemática. También existe consenso en la preocupación por la necesidad de estandarizar las dosis utilizadas, ya que cada autor defiende su aplicabilidad. Conclusión: A pesar de la falta de estandarización de parámetros para el tratamiento de heridas en general con estimulación eléctrica en ratas Wistar, los análisis morfológicos e histológicos muestran una respuesta efectiva en la reparación tisular en heridas sometidas a aplicaciones de microcorriente en diferentes períodos de tratamiento.
\end{abstract}

Palabras clave: Estimulación; Cicatrización de la herida; Wistar.

\title{
1. Introdução
}

A utilização da corrente elétrica com o objetivo de promover a cicatrização de feridas não é algo revolucionário ou novo, pois há registros de mais de 300 anos de sua utilização por meio de lâminas de ouro no tratamento de lesões cutâneas provocadas pela varíola (Guirro, 2002). Nos últimos anos, estudos melhor dirigidos e controlados foram realizados, a eletroterapia ganhou um novo impulso com a criação das "microcorrentes", inovando o conceito de tratamento a base de corrente elétrica e a forma de gerá-las. A corrente elétrica pode ser o gatilho que estimula a cura, o crescimento e a regeneração de todos os organismos vivos (Cheng et al., 1982). Foi demonstrado que em tecidos como os ossos, nervos e pele ocorrem alterações bioelétricas subsequentemente a uma lesão (Watson, 1995), tendo decrescido o fluxo elétrico na área lesada, diminuindo a capacitância celular e por consequência afetando a velocidade da reparação celular (Wolf, 1986). Assim, acredita-se que a correta aplicação das microcorrentes em um local lesionado aumenta o fluxo de corrente endógena, permitindo recuperar a sua capacitância, facilitando os transportes por meio da membrana, aumentando a síntese proteica e de ATP (Agne, 2004). Há incertezas ainda acerca dos mecanismos pelos quais a estimulação elétrica alcança seus resultados e se estes são significativos, embora claramente existam ligações que podem ser estabelecidas entre os efeitos hipotéticos do tratamento e o resultado da intervenção, segundo (Borges, 2006), a estimulação elétrica por meio de microcorrentes pode desencadear efeitos bioquímicos nos tecidos biológicos, podendo restabelecer a bioeletricidade do tecido, aumento da permeabilidade das membranas celulares e do transporte de aminoácidos além de auxiliar na síntese proteica. O objetivo deste artigo de revisão consistiu em avaliar a resposta cicatricial em ratos Wistar, tratados com as microcorrentes.

\section{Metodologia}

As seguintes bases eletrônicas de dados foram utilizadas, com seus respectivos períodos de busca: Medical Literature Analysis and Retrieval System Online (Medline) via Pubmed (1966 a janeiro de 2021) disponibilizada através do link: http://www.ncbi.nlm.nih.gov/pubmed/; (Scielo); e Literatura Latino-Americana e do Caribe em Ciências da Saúde (Lilacs), disponibilizada através do link http://regional.bvsalud.org/php/index.php (1982 a janeiro 2021). A busca resultou em estudos experimentais que abordassem a eletroestimulação no tratamento de feridas cutâneas em ratos wistar. A estratégia de busca utilizada foi a seguinte: a combinação dos termos "Stimulation", "wound healing" e "Wistar". 


\section{Seleção do estudo}

Inicialmente, objetivou-se fazer uma revisão sistemática com metanálise, no entanto, durante a estratégia de busca, observou-se que os diversos artigos publicados não foram realizados com a necessária homogeneidade nos padrões de técnicas utilizadas em seus experimentos, o que dificultou a comparação entre os artigos. Os parâmetros foram diferentes, tanto quanto as intensidades, frequências e tempo de aplicação utilizada, bem como os tipos de tecidos em que os experimentos foram realizados, tais como pele, tendões, músculos e ossos. Apesar disso, a estratégia de busca utilizada seguiu os parâmetros da sistematização.

Os autores leram os artigos selecionados na íntegra, de forma independente, baseando-se nos critérios de inclusão e exclusão. Quando a divergência persistia entre os autores, havia uma a discussão até o consenso. Os itens avaliados foram selecionados através dos resultados dos artigos revisados, determinados pela histologia e/ou pelos dados morfométricos. Para facilitar a compreensão dos estudos, um quadro com a extração dos dados foi elaborado pelos autores (Quadro 1).

Os critérios de inclusão consistiram em estudos com ratos wistar, tratados com eletroestimulação, publicados nas línguas inglesa ou portuguesa no período entre 1966 a 2021. Os critérios de exclusão consistiram em estudos com feridas infectadas, correntes de alta tensão, tratamento realizado no pré-operatório e estudos que não abordavam diretamente a aplicação da eletroestimulação no processo de cicatrização em feridas em wistar.

\section{Avaliação da qualidade}

Os critérios para avaliar a qualidade dos estudos seguiram as diretrizes do Arrive (Animal Research: Reporting of In Vivo Experiments). Link: http://www.elsevier.com/_data/promis_misc/622936arrive_guidelines.pdf. Foi avaliada, a qualidade metodológica, a integralidade do período de seguimento dos estudos, método de randomização e, mascaramento dos examinadores para os resultados. O domínio dos 3R Replacement (Substituição), substituir o método que utilizam animais vertebrados vivos e conscientes. Reduction (Redução), reduzir o número de animais utilizados para obter informação representativa e precisa. Refinement (Refinamento), qualquer desenvolvimento que leve a uma diminuição na severidade de processos cruéis aplicados aos animais utilizados.

\section{Resultados}

Ao final da estratégia de busca, foram identificados 26 artigos, depois de aplicados os critérios de inclusão e exclusão restaram sete artigos para serem lidos na íntegra. Respeitando os critérios de inclusão e, tendo seus dados sido analisados pormenorizadamente (Fluxograma 1). 
Research, Society and Development, v. 11, n. 1, e53311125295, 2022

(CC BY 4.0) | ISSN 2525-3409 | DOI: http://dx.doi.org/10.33448/rsd-v11i1.25295

Figura 1. Fluxograma dos artigos das bases eletrônicas de dados.

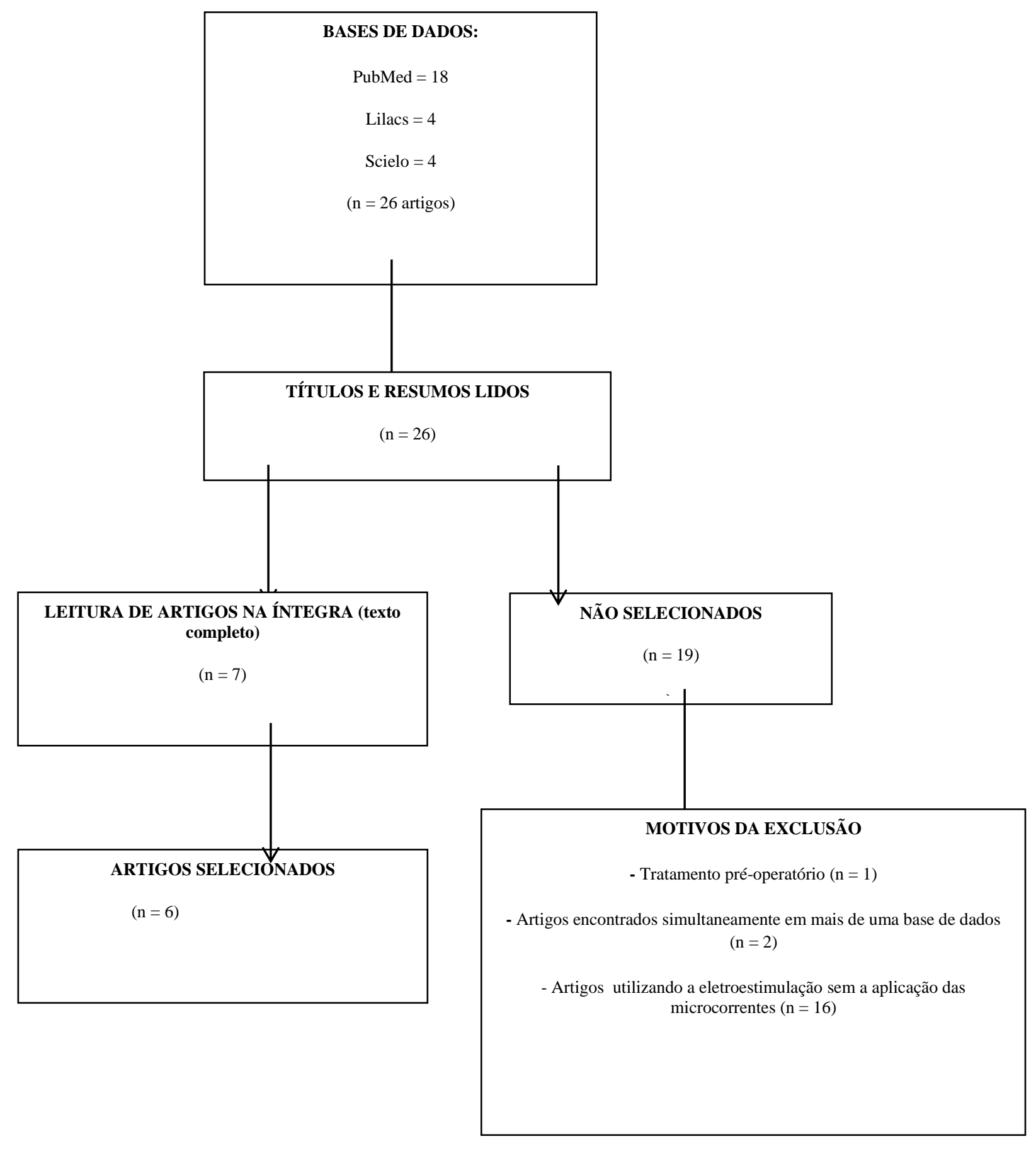

Fonte: Autores. 
Quadro 1: Síntese das evidências apresentadas pelos estudos.

\begin{tabular}{|c|c|c|c|c|}
\hline Autores & Amostra & Intervenção & Variáveis estudadas & Resultados \\
\hline (Castro et al., 2012) & $\begin{array}{c}\mathrm{N}=\text { Ausente } \\
\text { G6. }\end{array}$ & $\begin{array}{l}\text { Intensidade: } 10 \mu \mathrm{A} . \\
\text { Tempo: } 2 \mathrm{~min} / \mathrm{dia} . \\
\text { Período: } 10 \text { dias de } \\
\text { tratamento }\end{array}$ & $\begin{array}{l}\text { Leucócitos, } \\
\text { Fibroblastos, } \\
\text { hiperplasia, epitelização } \\
\text { e angiogênese. }\end{array}$ & $\begin{array}{l}\text { A cicatrização das feridas neste modelo } \\
\text { experimental em todos os parâmetros } \\
\text { do estudo }(\mathrm{P}<0,05) \text { quando comparado } \\
\text { ao grupo controle, com efeitos positivos } \\
\text { em tecido recém-formado, número de } \\
\text { vasos formados e porcentagem de fibras } \\
\text { colágenas maduras. }\end{array}$ \\
\hline $\begin{array}{c}\text { (Passarini Junior et } \\
\text { al., 2012) }\end{array}$ & $\begin{array}{c}\mathrm{N}=48 \text { ratos Wistar } \\
4 \mathrm{G}\end{array}$ & $\begin{array}{l}\text { Intensidade: } 10 \mu \mathrm{A} \\
\text { Tempo: } 2 \mathrm{~min} / \mathrm{dia} \\
\text { Período: } 14 \text { dias de } \\
\text { tratamento. }\end{array}$ & $\begin{array}{l}\text { Regeneração epitelial, } \\
\text { células inflamatórias, } \\
\text { formação de vasos e } \\
\text { fibroblastos. }\end{array}$ & $\begin{array}{l}\text { A reparação tecidual foi mais } \\
\text { expressiva nos animais tratados com a } \\
\text { MC, comparado com o GC, o teste } \\
\text { ANOVA ( }<<0,05) \text {, indica diferença } \\
\text { significativa entre diferentes períodos } \\
\text { de tempo. }\end{array}$ \\
\hline (Gaspi et al., 2011) & $\begin{array}{c}\mathrm{N}=36 \text { ratos wistar } \\
4 \mathrm{G} .\end{array}$ & $\begin{array}{l}\text { Intensidade: } 10 \mu \mathrm{A} \\
\text { Tempo: } 2 \mathrm{~min} / \mathrm{dia} \\
\text { Período: } 10 \text { dias de } \\
\text { tratamento }\end{array}$ & $\begin{array}{l}\text { Leucócitos, fibroblastos, } \\
\text { hiperplasia, epitelização, } \\
\text { angiogênese } \\
\text { reorganização tecidual. }\end{array}$ & $\begin{array}{l}\text { A aplicação da MC foi altamente eficaz } \\
\text { na termos dos parâmetros analisados ( } \mathrm{P} \\
<0,05) \text {, com efeitos positivos sobre a } \\
\text { área de tecido recém-formado, número } \\
\text { de fibroblastos, número de vasos } \\
\text { sanguíneos formados e espessura } \\
\text { epitelial. }\end{array}$ \\
\hline (Santos et al.; 2004) & $\begin{array}{c}\mathrm{N}=32 \text { ratos Wistar } \\
2 \mathrm{G} .\end{array}$ & $\begin{array}{l}\text { Intensidade: } 50 \mu \mathrm{A} \\
\text { Tempo: } 20 \mathrm{~min} / \mathrm{dia} . \\
\text { Período de } 21 \text { dias de } \\
\text { tratamento. }\end{array}$ & $\begin{array}{l}\text { Regeneração cicatricial, } \\
\text { fibrose, proliferação de } \\
\text { fibroblastos, } \\
\text { vascularização e } \\
\text { fibras colágenas. }\end{array}$ & $\begin{array}{l}\mathrm{Na} \text { microscopia foram observadas } \\
\text { alterações na quantidade e estrutura de } \\
\text { fibroblastos, e no colágeno do grupo } \\
\text { experimental, com diferença } \\
\text { estatisticamente significativa }(\mathrm{p}<0,01) \text {. }\end{array}$ \\
\hline $\begin{array}{c}\text { (Cardoso et al., } \\
\text { 2015) }\end{array}$ & $\mathrm{N}=108$ ratos Wistar. & $\begin{array}{l}\text { Intensidade: } 0,5 \mathrm{~mA} \\
\text { Tempo: } 20 \mathrm{~min} / \mathrm{dia} \\
\text { Periodo: } 21 \mathrm{dias} \mathrm{de} \\
\text { tratamento. }\end{array}$ & $\begin{array}{l}\text { Exsudato: } \\
\text { regeneração epitelial, } \\
\text { vascularização } \\
\text { fibrose. }\end{array}$ & $\begin{array}{l}\text { As variáveis apresntram diferença } \\
\text { significativa no dia } 7(\mathrm{p}<0,01) \text { grupo } \\
\text { MC comparado com grupo Sham. Não } \\
\text { houve diferença significativa no } \\
\text { período de tempo de } 14 \text { e } 21 \text { dias. }\end{array}$ \\
\hline
\end{tabular}

* $\mathrm{N}=$ (número de animais), ${ }^{*} \mathrm{G}=$ (número de grupos), $* \mathrm{MC}=$ (microcorrentes), ${ }^{*} \mathrm{GC}$ (grupo controle). Fonte: Autores.

\section{Discussão}

Os trabalhos encontrados mencionaram efeitos benéficos da eletroestimulação no processo cicatricial, contudo as metodologias utilizadas não seguem o Guidelines para os trabalhos com animais, nem descrevem com detalhes a metodologia utilizada para que esta seja reprodutível. Por exemplo, não dizem se realizaram o cálculo do tamanho da amostra nem como decidiram sobre a intensidade, a frequência ou o tempo de aplicação e esse fato dificulta a reprodutibilidade do método publicado.

Já em 1975, Konikoff et al. investigaram o uso da corrente elétrica na reparação óssea bem como, na reparação de feridas cutâneas. Nesse estudo foi realizada a aplicação da intensidade $20 \mu \mathrm{A}$ (microampère) em lesões de pele no dorso de coelhos durante sete dias. Os autores demonstraram uma melhor resposta na cicatrização das feridas estimuladas. Em 1843, Dubois-Reymond et al. relataram que a corrente com intensidade de $1 \mathrm{~mA}$ estimula a cicatrização de feridas.

Balakatounis e Angoules (2008), definiram a técnica de aplicação da microcorrente como sendo um corrente de intensidade menor ou igual a $1 \mathrm{~mA}(1000 \mu \mathrm{A})$, contudo, também não deixaram claro como definiram esse parâmetro. Segundos esses autores esse tipo corrente varia, de acordo com a intensidade, a frequência e o tempo de duração. A microcorrente produz uma baixa voltagem e a união desses parâmetros é capaz de gerar a eletroterapia. Afirmam que utilizar a 
frequências entre $0,3 \mathrm{~Hz}$ e $50 \mathrm{~Hz}$ mantem uma baixa voltagem na faixa de milliamperes, que não causa estímulos dolorosos, como também favorece a cicatrização. Estes trabalhos, nos permitem imaginar que os autores deveriam ter testado várias frequências e intensidades em muitos pacientes até definir qual delas não causava estímulos dolorosos, não sendo claros sobre esse aspecto, que nos parece extremamente relevante. Teriam eles escolhido números tão precisos aleatoriamente?

Já Alvarez et al. (1983). Estudaram o efeito da estimulação elétrica para cicatrização de pele em outros animais (porcos), tratados com intensidade de $50 \mu \mathrm{A}$ a $300 \mu \mathrm{A}$, do primeiro ao sétimo dia. Nesse estudo notou um aumento na capacidade de síntese de colágeno nos dias 5, 6 e 7 nas feridas tratadas com eletroestimulação. Assim como observaram a aceleração do processo de reparação tecidual.

Em 1994, Leffman et al. descreveram que modificaram o protocolo de tratamento descrito por Carley e Wainapel em 1985 para cicatrização de feridas em porcos, utilizando doses mais altas com um período de tempo superior, intensidade de $300 \mu \mathrm{A}$ durante 24 horas por dia. Estes autores concordaram com outros, definindo também que a estimulação por micro amperagem é considerada de baixa amperagem quando utilizada a intensidade e frequência $\left(<1 \mathrm{~mA} / \mathrm{cm}^{2}\right.$ e $\left.<3 \mathrm{~Hz}\right)$ para realização da terapêutica. Através desta definição Leffman et al. (1994) modificaram novamente os critérios de tratamento utilizando a intensidade de $100 \mu \mathrm{A}$, com frequência de $0,3 \mathrm{~Hz}$, por 2 horas dia, durante 14 dias de tratamento.

Byl et al. (1994) usaram a microcorrente para a cicatrização de feridas em porcos, com a intensidade de $100 \mu \mathrm{A}$, frequência de 0,1 Hz e tempo de aplicação diária de 1 hora. O tratamento seguiu o protocolo usado por Alvarez et al. (1983) que utilizou uma hora de tratamento por dia. Segundo os autores o tamanho dos eletrodos pode ter influenciado na passagem da corrente durante o tratamento, o que pode ter diminuído a acuidade da microcorrente. Defendendo que a microcorrente pode ter resultado positivo em humanos, pois, em estudo experimental não apresentou efeito na cicatrização de feridas.

Reger et al. (1999) avaliaram a cicatrização da ferida experimental, em 30 porcos submetidos a eletroestimulação. Dessa vez, as lesões foram estimuladas por 2 horas, durante 30 dias. A intensidade utilizada nesse estudo foi de $300 \mu \mathrm{A}$, com frequência de $40 \mathrm{~Hz}$. Os autores defendem que o protocolo parece orientar a nova formação de colageno na cicatrização de feridas em porcos.

O estudo de Demir et al. (2004), criaram um novo protocolo utilizando $300 \mu \mathrm{A}$ durante 30 minutos por dia, não descrevendo quantos $\mathrm{Hz}$ foram adotados, mesmo com inúmeros artigos mencionando a influência da frequência sobre os resultados do tratamento. Concluem que a eletroestimulação apresentou efeitos benéficos durante a fase inflamatória quando comparado ao grupo controle. Este trabalho utilizou animais diferentes de outros artigos, ou seja, fêmeas Swiss-Albino, tanto os parâmetros metodológicos diferentes, quanto as espécies diferentes também prejudica a comparação desses resultados com outros publicados, que utilizaram em sua maioria ratos Rattus novergicus (Wistar) machos.

Em estudo semelhante Taskan et al. (1997), utilizam $300 \mu \mathrm{A}$, durante 30 minutos. Estes autores também não descrevem se houve randomização da amostra. Foram utilizados neste estudo ratos fêmeos Sprague-Dawley. As fibras colágenas foram mais densas no grupo tratado quando comparo com o grupo SHAM.

Em nova metodologia, Santos et al. (2004) investigaram o efeito da eletroestimulação, com aplicação da microcorrente, dessa vez não utilizou feridas cirúrgicas, mas optaram por peeling com ácido na pele dos ratos 48 horas após a queimadura. $\mathrm{O}$ período de tratamento foi de 21 dias, com frequência de $0,5 \mathrm{~Hz}$, intensidade de $50 \mathrm{~mA}$ durante 20 minutos por dia. O tratamento proposto pelos autores demonstrou mudança no número de fibroblastos e fibras colágenas no grupo experimental.

Em 2009, Mendonça et al. publicaram um artigo utilizando o tratamento tópico com Aloe Vera e o uso da microcorrente para reparação tecidual. Apesar de seguirem o Guidelines, não descreveram o calculo do tamanho da amostra, estranhamente, esse trabalho não cita o número de animais por grupo, apenas que os animais foram randomizados, sem descrever por qual método. Relatam ainda que estes foram divididos em quatro grupos. Considerando que o processo de 
reparação tecidual tem início imediato, o tratamento poderia ter sido iniciado nas primeiras 24 horas. Segundo os autores a intervenção foi realizada durante 10 dias. $\mathrm{O}$ tratamento proposto por esses autores não seguem nenhum protocolo anterior e, não descrevem como chegaram a utilizar a intensidade de 10 Microampères, nem o porquê do tempo de tratamento de 2 minutos diários durante dez dias. A aplicação da microcorrente teve efeito positivo com os parâmetros utilizados, apresou nova formação tecidual mais cedo e, aumentou o número de fibroblastos e vasos formados.

Zuzzi et al. (2013), estudaram o uso da eletroestimulação na reparação da cartilagem xifoide, em Wistar machos. O tratamento utilizou a intensidade de $20 \mu \mathrm{A}$, frequência de $0,1 \mathrm{~Hz}$ por 5 minutos. Por serem diferentes, a metodologia e o tecido testado (cartilagem), também foram impeditivas de comparações com a maioria dos estudos que utilizaram pele.

Ciccone et al. (2013), também estudaram o efeito da microcorrente na reparação da cartilagem xifoide em Wistar, no entanto com outros critérios de frequência $(0,3 \mathrm{~Hz})$ e tempo de aplicação ( 5 minutos) diariamente durante 35 dias. Nesse artigo os autores afirmam que o grupo tratado teve fibras colágenas mais evidentes.

Passarini-Junior et al. Investigaram os efeitos do óleo da Jatropha curcas L. e a microcorrente em lesões experimentais de pele em ratos Wistar. Os autores seguiram o International regulations on animal testing and biodversity. Seguiram o protocolo de Medonça et al. que demonstraram resultado positivo no uso da microcorrente para o tratamento de feridas. Quarenta e oito ratos Wistar foram utilizados nesse estudo, cada grupo com doze animais. Diferente do estudo de (Mendonça et al., 2009) que não descreveu o número de animais, nem o número de grupos. No trabalho de Passarini-Junior et al. a intervenção teve início tardio (48 horas após a realização das feridas), o período tratamento foi de 14 dias. Não descreveram o método de randomização, bem como de que forma decidiram pelo tamanho da amostra.

Diferentemente de outros estudos que aguardaram 24 horas para iniciar o tratamento, Freitas et al., 2013, o iniciaram imediatamente após a lesão, com apliações diárias durante 10 dias. Intensidade de $160 \mu \mathrm{A}$ e frequência de $60 \mathrm{~Hz}$ durante 15 minutos por dia. O início do tempo de aplicação da eletroestimulção é considerado risco de viés, visto que, nas primeiras horas o processo de reparação tecidual já está acontecendo de forma natural, ou seja, fisiológica. Nesse estudo há ausência do cálculo do tamanho da amostra. Mencionam que foram utilizados 40 ratos Wistar, alocados aleatoriamente em quatro grupos com dez animais.

Castro et al. (2012) seguiram o international guidelines on animal experimentation and biodiversity, no entanto, a forma como foram selecionados os animais não é descrita, havendo um mascaramento na forma de seleção. O estudo tem ausência do cálculo do tamanho da amostra. Estes autores mencionam que foi escolhido o número dos grupos experimentais de acordo com a minimização do sofrimento dos animais, o que não conseguimos entender. Foram divididos em sete grupos com nove animais. Também optaram por iniciar o tratamento após 24 horas da lesão cutânea. Os parâmetros utilizados foram: intensidade de $10 \mu \mathrm{A}$, tempo de tratamento de 2 minutos por dia e frequência de 0,3 Hz. Descrevem que a escolha de dois minutos de aplicação seguia o protocolo de Mendonça et al. (2009).

Gaspi et al. (2011) seguiram o international rules considering the animal experiments and biodiversity righ. Comparam a utilização da microcorrente e a utilização do extrato da planta Oncidium flexuosum Sims na cicatrização de ferida cutâneas. A amostra foi de 36 animais, divididos através de randomização em quatro grupos com nove animais. $\mathrm{O}$ tratamento com a microcorrente utilizou a intensidade de $10 \mu \mathrm{A}$, tempo de 2 minutos dia. A intervenção iniciou 24 horas após a lesão e, o período de intervenção foi de 10 dias. Como os demais estudos há ausência do cálculo do tamanho da amostra, como o método de randomização e a escolha do tratamento proposto.

Observando os artigos descritos, verificamos que apesar da eletroestimulação ser descrita com resultados promissores, ainda encontra-se sem parâmetros padronizados, cada autor faz de um jeito, o que também leva os profissionais que a estão utilizando a seguir padrões distintos, não há, portanto, um protocolo proposto, mais detalhado. As doses têm sido escolhidas aleatória e empiricamente pela grande maioria dos autores, bem como o tempo de aplicação, a frequência utilizada (Hz) e as 
doses (Ampères). Os trabalhos experimentais existentes são em sua maioria em animais diferentes, o que exclui qualquer possibilidade de realização de uma revisão sistemática com metanálise, a qual seria um passo essencial para futuras tomadas de decisão clínica. Apesar do método já estar sendo amplamente utilizado em pacientes, ainda carecemos de novas pesquisas que possam comprovar os reais benefícios da eletroestimulação, que permitam a propositura de protocolos de tratamento nos diferentes tecidos. Ou seja, as feridas cicatrizariam apenas pelo fator tempo. Até que ponto os custos com aquisição de eletroestimuladores, com o tempo do profissional são válidas? Parece que tem sim o seu valor, mas com quantos Ampères? Com qual frequência? Com quanto tempo? Qual autor está certo? Qual a menor dose e tempo eficaz? Em quais tecidos o tratamento é mais eficiente? O que ocorreria em feridas infectadas? A nosso ver ainda restam muitas questões a ser respondidas e esperamos que este artigo suscite pesquisadores a respondê-las para que o tratamento com eletroestimulação que se mostra tão promissor, possa realmente contribuir da melhor forma e com o melhor custo-benefício.

\section{Conclusão}

Apesar da falta de padronização para o tratamento de feridas em Wistar, as análises morfológica e histológica, demonstram respostas eficazes no reparo tecidual nas feridas submetidas às aplicações das microcorrentes nos diferentes períodos de tratamento.

\section{Referências}

Agne, J. E. (2004). Eletrotermoterapia teoria e prática. Pallotti.

Alvarez, O. M., mertz, p.. M., smerbeck, r. V., \& eaglstein, w. H. (1983). The healing of superficial skin wounds is stimulated by external electrical current. Journal Of Investigative Dermatology, 81(2), 144-148. 10.1111/1523-1747.ep12543498. https://www.jidonline.org/article/s0022-202x(15)43152-5/pdf.

Balakatounis, K. C., \& Angoules, A. G. (2008). Low-intensity electrical stimulation in wound healing: Review of the efficacy of externally applied currents resembling the current of injury. Eplasty, 8, 283-291. https://www.ncbi.nlm.nih.gov/pmc/articles/PMC2396465/pdf/eplasty08e28.pdf.

Borges, F. S. (2006). Modalidades terapêuticas nas disfunções estéticas. Phorte.

Byl, N. N, McKenzie, A. L, West, J. M., Whitney, J. D., Hunt, T. K, Hopf, H. W., \& Scheuenstuhl, H. (1994). Pulsed microamperage stimulation: a controlled study of healing of surgically induced wounds in Yucatan pigs. Phsical Therapy, 74(3), 201-213. 10.1093/ptj/74.3.201. https://pubmed.ncbi.nlm.nih.gov/8115454.

Cardoso, M. P., Albuquerque, A. O., Girão, V. C., Pompeu, M. M., Silva, C. I., Azevedo, O. G., Guimarães, S. B., \& Vasconcelos, P. R. (2015). Preconditioning with mono and polyunsaturated fatty acids and low-intensity electrical stimulation. Effects on skin repair in rats. Acta Cirúrgica Brasileira., 30 (2), 107-114. 10.1590/S0102-86502015002000004. http://www.scielo.br/pdf/acb/v30n2/0102-8650-acb-30-02-00107.pdf.

Carley, P. G., \& Wainapel, S. F. (1985). Electrotherapy for accelerations of wound healing: Low intensity direct current. Archives of Physical Medicine and Rehabilitation, 66(7), 443-446. https://pubmed.ncbi.nlm.nih.gov/3893385.

Castro, F. C.; Magre, A.; Cherpinski, R.; Zelante, P. M.; Neves, L. M.; Esquisatto, M. A.; Mendonça, F. A., \& Santos, G. M. (2012). Effects of microcurrent application alone or in combination with topical Hypericum perforatum L. and Arnica montana L. on surgically induced wound healing in Wistar rats. Homeopathy, 101(3), 147-153. 10.1016/j.homp.2012.05.006. http://www.homeopathyjournal.net/article.

Cheng, N., Van Hoof, H., Bockx, E., Hoogmartens, M. J., Mulier, J. C., De Dijcker, F. J., Sansen, W. M., \& De Loecker, W. (1982). The effects of electrical currents on ATP regeneration, protein synthesis, and membrane transport in rat skin. Clinical Orthopedics and Related Research, (171), 264-272, 1982. http://www.ncbi.nlm.nih.gov/pubmed.

Ciccone, Cde C., Zuzzi, D. C., Neves, L. M. G., Mendonça, J. S., Joazeiro, P. P., \& Esquisatto, M. A. M. (2013). Effects of microcurrent stimulation on hyaline cartilage repair in immature male rats (Rattus novergicus). BMC Complementary and Alternative Medicine, 13, 1-9. 10.1186/1472-6882-13-17. https://www.ncbi.nlm.nih.gov/pmc/articles/PMC3554581/pdf/1472-6882-13-17.pdf.

Demir, H., Balay,, H. \& Kirap, M. (2004). A comparative study of the effects of electrical stimulation and laser treatament on experimental wound healing in rats. Journal of Rehabilitation Research \& Development, $41(2), \quad 147-154 . \quad 10.1682 / \mathrm{jrrd} .2004 .02 .0147$. https://www.rehab.research.va.gov/jour/04/41/2/pdf/Demir.pdf

Freitas, R. P., Macedo, A. B., Oliveira, A. R., Ramos, A. M., \& Vieira, W. H. (2014). Comparative study of Low-level laser therapy and icrocurrent on the healing of skin burns in rats. Acta Scientiarum. Health Sciences, 36(1),5-10. 10.4025/actascihealthsci.v36i1.14668. https://periodicos.uem.br/ojs/index.php/ActaSciHealthSci/article/view/14668.

Gaspi. F. O., Foglio, M. A., Carvalho, J. E., Santos, G. M., Testa, M., Passarini JR, J.R., Moraes, C. P., Esquisatto, M. A., Mendonça, J. S., \& Mendonça, F. A. (2011). Effects of the Topical Aplication of Hydroalcohlic Leaf Extract of Oncidium flexuosum Sims. (Orchidaceae) and Microcurrent on the Healing of 
Research, Society and Development, v. 11, n. 1, e53311125295, 2022

(CC BY 4.0) | ISSN 2525-3409 | DOI: http://dx.doi.org/10.33448/rsd-v11i1.25295

Wounds Surgically Imduced in Wistar Rats. Evidence-Based Complementary and Alternative Medicine. 10.1155/2011/950347. http://www.hindawi.com/journals/ecam/2011/950347/.

Guirro, E. (2002). Fisioterapia dermato-funcional. fundamentos, recursos, patologias (3rd ed.). Manole.

Konikoff, J. J. (1976). Eletrical promotion of soft tissue repairs. Annals of Biomedical Engineering, 4(1), 1-5. 10.1007/BF02363553. http://www.ncbi.nlm.nih.gov/pubmed.

Leffmann, D. J., Arnall, D. A., Holmgren, P. R., \& Cornwall, M. W. (1994). Effect of microamperage stimulation on the rate of wound healing in rats: A histological study. Phsical therapy, 74(3), 195-200. 10.1093/ptj/74.3.195. https://pubmed.ncbi.nlm.nih.gov/8115453

Medonça, F. A., Passarini Junior, J. R., Esquisato, M. A., Mendonça, J. S., Franchini, C. C., \& Santos, G. M. (2009). Effects of the application of Aloe vera (L.) and microcurrent on the healing of wounds surgically induced in Wistar rats. Acta Cirúrgica Brasileira, 24(2), 150-155. 10.1590/s010286502009000200013. https://www.scielo.br/j/acb/a/BsJkSzTpcFWtLvg84Pf6FTh/?format=pdf\&lang=en.

Passarini-Junior, J. R., Gaspi, F. O., Neves, L. M., Esquisatto, M. A., Santos, G. M., \& Mendonça, F. A. (2012). Aplication of Jatropha curcas L. seed oil (Euphorbiaceae) and microcurrent on the healing of experimental wounds in wistar rats. Acta Cirúrgica Brasileira, 27(7), 441- 447. 10.1590/s010286502012000700002. https://www.scielo.br/j/acb/a/Bypd8CWjJkfH3Nzg9whLpnS/?format=pdf\&lang=en.

Reger, I. S., Hyodo, A., Negami, S., Kambic, H. E., \& Sahgal, V. (1999). Experimental wound healing with electrical stimulation. Blackwell Science, 23(5), 460-462. 10.1046/j.1525-1594.1999.06365.x. https://pubmed.ncbi.nlm.nih.gov/10378943

Santos, V. N., Ferreira, L. M., Horibe, E. K., Duarte. I. S. (2004). Electric Microcurrent in the Restoration of the Skin Undergone a Trichloroacetic Acid Peeling in Rats. Acta Cirurgica Brasileira, 19(5), 466-470. doi.org/10.1590/S0102-86502004000500003. http://www.scielo.br/pdf/acb/v19n5/a03v19n5.pdf.

Taskan, I., Ozyazgan, I., Tercan, M., Kardas, Y., Balkanli, S., Saraymen, R., Zorlu, U., \& Ozugul, Y. (1997). A comparative study of the effect of ultrasound and electrostimulation on wound healing in rats. Plastic and Reconstructive Surgery, 100(4), 966-972. 10.1097/00006534-199709001-00020. http://www.ncbi.nlm.nih.gov/pubmed.

Watson T. (1994). The bioelectric correlates of musculoskeletical injury and repair https://www.proquest.com/openview/95ecf37455def143d286e4897702 6df3/1?pq-origsite $=$ gscholar \&cbl=2026366\&diss $=y$.

Wolf, A. A. Injury currents: Their electrical and mathematical properties in wound healing. Proceedings of the 12th Northeast Bioengineering Conference, New York, NY, IEEE ..., 1986. 4, 1986.

Zuzzi, D. C., Ciccone, Cde C., Neves, L. M., Mendonça, J. S,, Joazeiro, P. P., \& Esquisatto, M. A.(2013). Evaluation of the effects of electrical stimulation on cartilage repair in adult male rats. Tissue and Cell, 45(4), 275-281. 10.1016/j.tice.2013.02.003. http://www.ncbi.nlm.nih.gov/pubmed. 